Pacific Journal of Mathematic 


\title{
SEMIVARIETIES AND SUBFUNCTORS OF THE IDENTITY FUNCTOR
}

\author{
S. A. HuQ
}

We study certain subcategories called semivarieties and obtain Kaplanasky's theorem on the decomposition of Abelian groups into a divisible group and a reduced group under the frame-work of category theory; We also investigate the connection of these epicoreflective subcategories with varieties.

Semivarieties are subcategories of a category with cartain axioms; such subcategories play an important role in Abelian categories and a description of these by means of coreflection, appears in the general context in the work of Mitchell [8, $\S 5, \S 6$, III]. Broader classes than these have also been studied by Amitsur [1], Carreau [2], under the title of $H I-R I$ properties of radicals and their classes. Our aim in this note is to give a categorical proof of Kaplanasky's Theorem $3[6, \S 5]$ and while so doing we generalize the concepts of varieties and variety functors of Fröhlich [4] under abstract frame work utilizing Maranda's [9] concept of a radical.

$\mathscr{C}$ is a category equipped with the following axioms:

I. $\mathscr{C}$ has a null object.

II. Every morphism $\alpha$ in $\mathscr{C}$, admits a factorization $\alpha=\nu \mu$, where $\nu$ is a normal epimorphism and $\mu$ is a monomorphism; we are writing the composition in the precise way $\underbrace{\stackrel{\alpha}{\rightarrow} \cdot \vec{\beta}}_{\alpha \beta}$; where the dots
are the unnamed objects.

III. Every family of objects has a direct and a free product.

IV. The subobjects and factor objects of any objects form a set.

It is immediate that $\mathscr{C}$ admits null morphisms. The image of a morphism in $\mathscr{C}$, defined in axiom II and some time written as $(\nu, L, \mu)$ in the factorization $\stackrel{\nu}{\longrightarrow} \stackrel{\mu}{\longrightarrow} \cdot$, is uniquely determined to within equi-

valence. Every family of subobjects of an object $A$ in $\mathscr{C}$ has a union and as such every morphism has a kernel. Dual consideration holds for factor objects and cokernel. A map admitting null subobject as the kernel is a monomorphism and a sequence

$$
0 \longrightarrow A \stackrel{\alpha}{\longrightarrow} B \stackrel{\beta}{\longrightarrow} C \longrightarrow 0
$$

is exact if and only if $\alpha$ is a monomorphism, $\beta$ is a normal epimorphism and the subobject $(A, \alpha)$ serves as the kernel of $\beta$. For details on the notation used and results mentioned in this section the reader 
is referred to [7], [10]. In view of axiom II, one can check that every retraction is a normal epimorphism as such a monomorphism which is a retraction is invertible, [7] and further the composition of two normal epimorphisms is again normal. Also if $\mu$ is a normal monomorphism admitting cokernel $\nu$, then $\mu$ is the kernel of $\nu$ and dual result holds for the normal epimorphism $\nu$, admitting kernel $\mu$.

2. Subfunctors of $\mathrm{I}$. We shall consider covariant functors from $\mathscr{C} \rightarrow \mathscr{C}$, and such subfunctors of the identity functor $I$.

Definition. A subfunctor $(F, \mu)$ of $I$ will be called a normal subfunctor if for each $A \in \mathscr{C}$, the natural map $\mu_{A}: F(A) \rightarrow A$ is a normal monomorphism; and $(F, \mu)$ is called injective if $\mu_{A}$ is a coretraction.

A subfunctor $(F, \mu)$ of $\mathscr{C}$ is said to be a radical subfunctor, (dually coradical subfunctor) in the sense of Maranda [9] if for any $A,\left(F(A), \mu_{A}\right)$ has cokernel $(\varepsilon, C)$ then $1_{F^{\prime}(C)} \sim \omega\left[\omega=\omega_{A B}\right.$ always stands for the null morphism of $H(A, B)$ ], so that $F(\varepsilon): F(A) \rightarrow F(C)$ is the null normal epimorphism $\omega_{F^{(A), 0}}$ (dually for the inclusion $\mu_{A}: F(A) \rightarrow$ $A ; F\left(\mu_{A}\right): F F(A) \rightarrow F(A)$ is an equivalence).

3. Semivarieties and cosemivarieties. A semivariety $\mathscr{B}$ is a full subcategory of $\mathscr{C}$, satisfying the following axioms:

I. $f: A \rightarrow B$ is a monomorphism and $B \in B \Rightarrow A \in B$.

II. If $\left(A_{i}\right)_{i \in I}$ is an indexed set of objects of $\mathscr{B}$, then their direct product $\Pi A_{i} \in \mathscr{B}$.

Examples. (i) All varieties [4].

(ii) Torsion free groups (respectively Abelian groups) form a semivariety of all groups (respectively of Abelian groups)

(iii) Reduced groups form a semivariety of all Abelian groups.

(iv) Nilpotent free rings (i.e., a ring in which $a^{2}=0 \Rightarrow a=0$ ) in the category of rings.

Proposition 3.1. (1) If $(V, \mu)$ is a subfunctor of $I$, then the objects $A$ for which $\left(V(A), \mu_{A}\right) \sim(0, \omega)$ form a semivariety $\mathscr{B}_{V}$.

(2) If $\mathscr{B}$ is a semivariety then every object $A$ has a minimal normal subobject $\left(V_{.}(A), \mu_{\mathscr{B}}(A)\right)$ whose cokernel lies in $\mathscr{B}$. These $\left(V_{\mathscr{B}}(A), \mu_{\mathscr{O}}(A)\right)$ determine a normal radical subfunctor $\left(V_{\mathscr{B}}, \mu_{\mathscr{B}}\right)$ of $I$.

(3) If $V \sim V_{\mathscr{B}}$ then $\mathscr{B}=\mathscr{B}_{V}$. Conversely $\mathscr{B}=\mathscr{B}_{V}$ implies $V$ is a subfunctor of $V_{\mathscr{S}}$; but if $(V, \mu)$ is normal and a radical subfunctor then $\mathscr{B}=\mathscr{B}_{V} \Rightarrow V \sim V_{\mathscr{B}}$.

Proof. (1) Let $\mathscr{B}_{V}=\left\{A \mid\left(V(A), \mu_{A}\right) \sim(0, \omega)\right\}$ and all their mor- 
phisms. Since $(V, \mu)$ is a subfunctor of $I, 0 \in \mathscr{B}_{V}$. Thus $\mathscr{B}_{V}$ is a full subcategory.

Next if $A \stackrel{f}{\rightarrow} B$ is a monomorphism, then $V(f): V(A) \rightarrow V(B)$ is a monomorphism. Thus $B \in \mathscr{B}_{V} \Rightarrow V(B) \sim 0$ and as such $1_{V(A)}=\omega$ which implies $A \in \mathscr{B}_{V}$.

Next if $\left(A_{i}\right)_{i \in I}$ is a family of objects of $\mathscr{B}_{V}$, then we have a commutative diagram

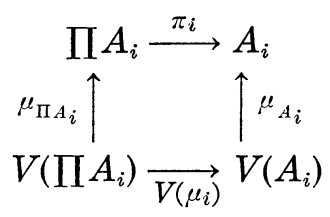

where the top horizontal is the natural projection for each $i$ which is a normal epimorphism. Now $A_{i} \in \mathscr{B}_{V} \Rightarrow\left(V\left(A_{i}\right), \mu_{A_{i}}\right) \sim(0, \omega)$ that is $\mu_{\Pi_{A} A_{i}} \pi_{i}=\omega$, i.e., $\mu_{\Pi_{A_{i}}}=\omega$ so $1_{V}\left({ }_{\Pi A_{i}}\right)=\omega$ giving $\Pi A_{i} \in \mathscr{B}_{V}$.

(2) Suppose $\mathscr{B}$ is a semivariety. We consider all normal subobjects $\left(V_{i}(A), \mu_{i}(A)\right)$ of $A$ whose cokernel $\left(\varepsilon_{i}, C_{i}\right)$ lies in $\mathscr{B}$. Then $\varepsilon_{i}: A \rightarrow C_{i}$ determine a unique map $\varepsilon: A \rightarrow \Pi C_{i}$ such that $\varepsilon \pi_{i}=\varepsilon_{i}$ (where $\Pi C_{i} \stackrel{\pi i}{\rightarrow} C_{i}$, stands for the direct product). Now $\Pi C_{i} \in \mathscr{B}$. We denote by $\left(V_{\mathscr{B}}(A), \mu_{\mathscr{B}}(A)\right)$ the kernel of $\varepsilon$; then this determines a normal subfunctor $\left(V_{\mathscr{B}}, \mu_{\mathscr{B}}\right)$ of $I$. We note $\left(V_{\mathscr{B}}(A), \mu_{\mathscr{B}}(A)\right)$ is also the kernel of the epimorphism $\nu$, in the image $(\nu, C, \mu)$ of $\varepsilon$. Then $\left(V_{\mathscr{A}}(A)\right.$, $\left.\mu_{3}(A)\right)$ is minimal normal subobject (in the sense of partial ordering of subobjects, see [7]) whose cokernel lies in $\mathscr{B}$ is obvious from the construction. Evidently $V_{\mathscr{B}}$ has the radical property.

(3) Suppose $\mathscr{B}$ is a semivariety and $V \sim V_{\mathscr{B}}$, then $\mathscr{B}_{V}$ consists of all objects $A$ for which $\left(V(A), \mu_{A}\right) \sim(0, \omega)$. Now $\mathscr{B}$ consists of all objects $A$ whose cokernel is $\left(1_{A}, A\right)$; thus $\mathscr{B}=\mathscr{B}_{V}$.

Now suppose $\mathscr{B}=\mathscr{B}_{V}$. If for any object $A, V(A) \stackrel{\mu_{A}}{\rightarrow} A$ and $V_{\mathscr{B}}(A) \stackrel{\mu_{\infty}}{\longrightarrow} A$ admit the cokernels $\left(\varepsilon^{*}, D^{*}\right)$ and $(\varepsilon, C)$ respectively then $C \in \mathscr{B}=\mathscr{B}_{V}$, i.e., $\left(V(C), \mu_{C}\right) \sim(0, \omega)$ so $\mu_{A} \varepsilon=\omega$, therefore there exists a monomorphism $\lambda_{A}: V(A) \rightarrow V_{\mathscr{D}}(A)$ such that $\lambda_{A} \mu_{\mathscr{B}}=\mu_{A}$. That this $\lambda$ is a functor monomorphism is easy to check. If further $V$ is normal and a radical subfunctor, then for the cokernel $\left(\varepsilon^{*}, D^{*}\right)$, $1_{V\left(D^{*}\right)} \sim \omega$, i.e., $D^{*} \in \mathscr{B}_{V}=\mathscr{B}$. Thus $\left(\varepsilon^{*}, D^{*}\right) \leqq(\varepsilon, C)$, i.e., $\varepsilon^{*} \sim \varepsilon$, giving $(V, \mu) \sim\left(V_{\mathscr{S}}, \mu_{\mathscr{A}}\right)$.

Dual to the concept of a semivariety is that of a cosemivariety.

Definition. A full subcategory $\mathscr{F}$ of $C$ is called a cosemivariety if

I. $f: A \rightarrow Q$ a normal $^{1}$ epimorphism $A \in \mathscr{F}, \Rightarrow Q \in \mathscr{F}$.

\footnotetext{
1 This is required since axiom II, is not self dual.
} 
II. $\left(A_{i}\right)_{i \in I}$ is a family of objects of $\mathscr{F}$, then their free product $\Pi^{*} A_{i} \in \mathscr{F}$.

ExAMPles. (i) All covarieties. In particular in the category of Abelian groups, torsion groups from a covariety.

(ii) Divisible groups form a cosemivariety of all Abelian groups. Mirror image of Proposition 3.1. will be

Proposition 3.1*. (1) If $F$ is subfunctor of the identity functor $I$ of $\mathscr{C}$, then the collection of all objects $A$ for whjch $\left(F(A), \mu_{A}\right) \sim$ $\left(A, 1_{A}\right)$ from a cosemivariety $\mathscr{F}^{F}$.

(2) If $\mathscr{F}$ is a cosemivariety, then every object $A$ has a maximal subobject $\left(F^{\widetilde{T}}(A), \mu^{\sigma}(A) \in \mathscr{F}\right.$. This $\left(F^{\sigma}, \mu^{\sigma}\right)$ is a coradical subfunctor of $I$.

(3) $F \sim F^{\sigma} \Rightarrow \mathscr{F}=\mathscr{F}^{F}$. Conversely $\mathscr{F}=\mathscr{F}^{F}$ implies $F^{-}$ is a subfunctor of $F$; further if $F$ is a coradical subfunctor then $\mathscr{F}=\mathscr{F}^{F} \Rightarrow F \sim F$.

We exhibit the proof for (1), leaving the second and third part for the reader to dualize their counter parts in Proposition 3.1 in the same way.

Let $\mathscr{F}^{F}=\left\{A \mid\left(F(A), \mu_{A}\right) \sim\left(A, 1_{A}\right)\right\}$ and all their morphisms; the null object is obviously $\in \mathscr{F}^{F}$.

Next if $A \stackrel{\alpha}{\rightarrow} B$ is a normal epimorphism and $A \in \mathscr{F}^{F}$, then we have a commutative diagram

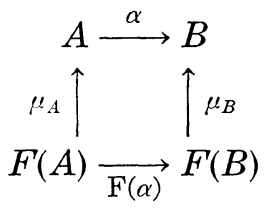

in which $\mu_{A}$ is an equivalence. Now $\mu_{A} \alpha$ is a normal epimorphism as observed in $\S 1$. Let $\delta$ be its kernel. Then $\delta F(\alpha)=\omega$, i.e., $F(\alpha)=$ $\mu_{A} \alpha \mu\left(\mu_{A} \alpha\right.$ is normal and therefore cokernal of $\left.\delta\right)$. Now $\mu_{A} \alpha \mu^{\prime} \mu_{B}=$ $F(\alpha) \mu_{B}=\mu_{A} \alpha$. Thus $\mu \mu_{B}=1$, i.e., $\mu_{B}$ is a retraction and hence invertible, i.e., $B \in \mathscr{F}^{F}$.

Next suppose $\left(A_{i}\right)_{i \in I}$ be a family of objects of $\mathscr{C}$ belonging to $\mathscr{F}$, then we have for each $i$, a commutative diagram

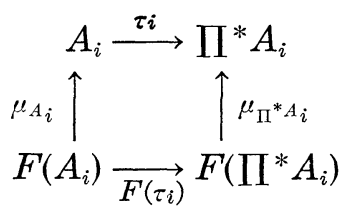

where $\tau_{i}$ are the natural monomorphisms associated with the free 
product. Now $\mu_{A_{i}}$ is invertible, let $\mu_{A_{i}}^{-1}$ be its inverse. Then $\mu_{A_{i}}^{-1} F\left(\tau_{i}\right): A_{i} \rightarrow F\left(\Pi^{*} A_{i}\right)$ determines a unique $\lambda: \Pi^{*} A_{i} \rightarrow F\left(\Pi^{*} A_{i}\right)$ such that $\tau_{i} \lambda=\mu_{A_{i}}^{-1} F\left(\tau_{i}\right)$.

Now $\tau_{i}\left(\lambda \mu_{\Pi^{*} A_{i}}\right)=\mu_{A_{i}}^{-1} F\left(\tau_{i}\right) \mu_{\Pi^{*} A_{i}}=\mu_{A_{i}}^{-1} \mu_{A_{i}} \tau_{i}=\tau_{i}$.

Thus $\lambda \mu_{\Pi^{*} A_{i}}=1$ showing that $\mu_{\Pi^{*} A_{i}}$ is a retraction and hence invertibe, i.e., $\Pi^{*} A_{i} \in \mathscr{F}^{F}$.

Thus if $(F, \mu)$ is any subfunctor of $I$, determining a semivariety $\mathscr{B}_{F}$ and a cosemivariety $\mathscr{F}^{F}$, then we have a functor transformation

$$
F^{\mathscr{F} F} \rightarrow \stackrel{\delta}{-} F \rightarrow \stackrel{\lambda}{-} F_{\mathscr{C}_{F}}, \text { and }
$$

for any object $A$, we have a commutative diagram

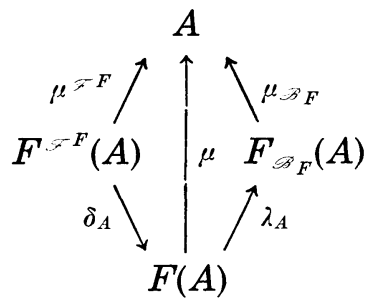

where $\mu_{\mathscr{B}_{F}}$ is a normal monomorphism.

It is clear from our construction that if $\mathscr{F}$ is a cosemivariety determining a normal radical subfunctor $\left(F^{\mathscr{F}}, \mu^{\sigma}\right)$ of $I$, then for any $A \in \mathscr{C}$ the cokernel of $\mu_{A}: F^{\mathscr{T}}(A) \rightarrow A \in \mathscr{B}_{F}{ }^{\sigma}$, and by Proposition 3.1 $F^{\mathscr{T}} \sim F_{\mathscr{S} F^{\mathscr{V}}}$, and we collect these informations in the following.

Proposition 3.2. If $\mathscr{F}$ is a cosemivariety, such that the functor $\left(F^{\sigma}, \mu^{\sigma}\right)$ determined by it is a normal radical subfunctor, then for any object $A$, we have the exact sequence

$$
0 \longrightarrow F^{\sigma}(A) \stackrel{\mu_{A}}{\longrightarrow} A \stackrel{\varepsilon}{\longrightarrow} C \longrightarrow 0
$$

where $F^{\mathscr{F}}(A) \in \mathscr{F}$ and $C \in \mathscr{\mathscr { B }}_{F}^{\mathscr{F}}$.

We are content to leave the reader to mirror the images of the above observations.

If $\mathscr{C}$ is the category of Abelian groups, and $\mathscr{F}$ is the cosemivariety of torsion groups (or divisible groups) then if to each group $A$, we assign the torsion subgroup $F^{\sigma}(A)$ (or the miximal divisible subgroup $F^{\widetilde{T}}(A)$ ), then $A / F^{\mathscr{T}}(A)$ lies in the semivariety of torsion free groups (or reduced groups).

Proposition 3.3. If $\mathscr{A}$ is an Abelian category in which a cosemivariety $\mathscr{F}$ determines a radical which is injective, then every object $A$ is the direct sum of an object in $\mathscr{F}$ and $\mathscr{B}_{F} \mathscr{F}$, and as 
such the identity functor splits having components in the cosemivariety and the semivariety.

Proof. We have an exact sequence $0 \rightarrow F^{\Im}(A) \stackrel{\mu}{\rightarrow} A \rightarrow A /_{F^{\mathscr{F}}(A)} \rightarrow 0$ in which the natural injection is a coretraction. Thus there exists a $\delta: A \rightarrow F^{\prime}(A)$ such that $\mu \delta=1$. So the theorem is obvious from Theorem 2.68 [5]. Thus for the category of abelian groups, if $F^{-}$ is the functor determined by the cosemivariety of divisible groups, then we have Theorem $3 .[6, \S 5]$.

It is easy to see that the variety functors (covariety functors or torsion radicals) [[4], [9]] are indeed radical functors (coradical functors) and a coradical subfunctor $(F, \mu)$ determines a cotriple $\left[(F \mu)^{-1}\right.$, $\mu, F]$ in the sense of Eilenberg and Moore [3] where $F \mu$ is the equivalence $F^{2} \rightarrow F$ which appears in the definition of coradical subfunctor given by $F \mu_{A}=F\left(\mu_{A}\right)$ for any object $A$ of $\mathscr{C}$, and this is cogenerated by the adjoint $F$ of the inclusion of the associated cosemivariety $\mathscr{F}=\mathscr{F}^{F}$ into $\mathscr{C}$, where $F$ can be considered as well a functor from $\mathscr{C} \rightarrow \mathscr{C}$ dropping the inclusion completely.

We notice if $V$, the normal radical functor associated with the semivariety $\mathscr{B}$ is a variety functor, then $\mathscr{B}$ is a variety; converse association holds, i.e, the normal radical functor associated with a variety will be a variety functor (11) in categories with the additional axiom.

(v) If $\alpha$ is a monomorphism and $\beta$ is a normal epimorphisms such that $\alpha \beta$ admits imags $\nu \mu$, then $\alpha$ normal $\Rightarrow \mu$ is normal.

Added in proof. While this work was in press, the author was given to understand by $\mathrm{P}$. Lecouturier that certain generalization of Fröhlich's work (a weaker version of Proposition 3.1) has also been obtained by him in more restricted class of categories (in which every epimorphism is normal, etc). However he does not obtain the characterization of semivarieties by normal radical functors.

\section{REFERENCES}

1. S. A. Amitsur, General theory of radicals, Amer. J. Math. 76 (1954), 100-125.

2. F. Carreau, Théorie générale des radicaux, Doctoral dissertation, Univ. de Montréal 1967.

3. Eilenberg and Moore, Adjoint functors and triples, Illinois J. Math. (2) 9, 381-398.

4. A. Frohlich, On groups over a D.G. near ring II; Categories and functors, Quart.

J. Math. Oxford (2) 11 (1960), 211-228.

5. P. Freyed, Abelian categories, Harper and Row, 1964.

6. I. Kaplanasky, Infinite abelian groups, Univ. of Michigan Press, Ann Arbor, 1956.

7. A. G. Kurosh, A. Kh. Livshits and E. G. Schulgeifer, The foundations of the theory of categories, Russ. Math. Surveys 15 (1960), 1-46.

8. B. Mitchell, Theory of categories, Academic Press, 1965. 
9. J. M. Maranda, Injective structures, Trans. Amer. Math. Soc. 110 (1964), 98-135. 10. M. S. Tsalenko, On the foundation of the theory of categories, Russ. Math. Surveys 15 (1960), 47-51.

11. S. A. Huq, Commutator, nilpotency and solvability in categories, Quart. J. of Math. (Oxon) 2, 19 (1968), 363-389.

Received February 23, 1968. Supported by a Postdoctoral fellowship in the Université de Montreal.

UNIVERSity of Montreal 



\section{PACIFIC JOURNAL OF MATHEMATICS}

\section{EDITORS}

H. ROYDEN
Stanford University
Stanford, California

\section{R. R. PHELPS}

University of Washington

Seattle, Washington 98105
J. DUGUNDJI

Department of Mathematics

University of Southern California

Los Angeles, California 90007

\section{RICHARD ARENS}

University of California

Los Angeles, California 90024

\section{ASSOCIATE EDITORS}

E. F. BeCKenbaCh
B. H. NEUMANN

F. WOLF
K. YoshidA

\section{SUPPORTING INSTITUTIONS}

\author{
UNIVERSITY OF BRITISH COLUMBIA \\ CALIFORNIA INSTITUTE OF TECHNOLOGY \\ UNIVERSITY OF CALIFORNIA \\ MONTANA STATE UNIVERSITY \\ UNIVERSITY OF NEVADA \\ NEW MEXICO STATE UNIVERSITY \\ OREGON STATE UNIVERSITY \\ UNIVERSITY OF OREGON \\ OSAKA UNIVERSITY \\ UNIVERSITY OF SOUTHERN CALIFORNIA
}

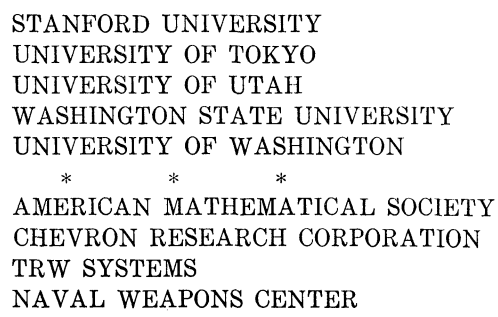

The Supporting Institutions listed above contribute to the cost of publication of this Journal, but they are not owners or publishers and have no responsibility for its content or policies.

Mathematical papers intended for publication in the Pacific Journal of Mathematics should be in typed form or offset-reproduced, double spaced with large margins. Underline Greek letters in red, German in green, and script in blue. The first paragraph or two must be capable of being used separately as a synopsis of the entire paper. It should not contain references to the bibliography. Manuscripts, in duplicate if possible, may be sent to any one of the four editors. Please classify according to the scheme of Math. Rev. 36, 1539-1546. All other communications to the editors should be addressed to the managing editor, Richard Arens, University of California, Los Angeles, California, 90024.

50 reprints are provided free for each article; additional copies may be obtained at cost in multiples of 50 .

The Pacific Journal of Mathematics is published monthly. Effective with Volume 16 the price per volume (3 numbers) is $\$ 8.00$; single issues, $\$ 3.00$. Special price for current issues to individual faculty members of supporting institutions and to individual members of the American Mathematical Society: $\$ 4.00$ per volume; single issues $\$ 1.50$. Back numbers are available.

Subscriptions, orders for back numbers, and changes of address should be sent to Pacific Journal of Mathematics, 103 Highland Boulevard, Berkeley, California, 94708.

PUBLISHED BY PACIFIC JOURNAL OF MATHEMATICS, A NON-PROFIT CORPORATION

Printed at Kokusai Bunken Insatsusha (International Academic Printing Co., Ltd.), 7-17, Fujimi 2-chome, Chiyoda-ku, Tokyo, Japan. 


\section{Pacific Journal of Mathematics \\ Vol. 29, No. $2 \quad$ June, 1969}

Bruce Langworthy Chalmers, On boundary behavior of the Bergman kernel function and related domain functionals ................... 243

William Eugene Coppage, Peirce decomposition in simple Lie-admissible power-associative rings .............................. 251

Edwin Duda, Compactness of mappings...................... 259

Earl F. Ecklund Jr., On prime divisors of the binomial coefficient......... 267

Don E. Edmondson, A modular topological lattice ............... 271

Phillip Alan Griffith, A note on a theorem of Hill ................... 279

Marcel Herzog, On finite groups with independent cyclic Sylow

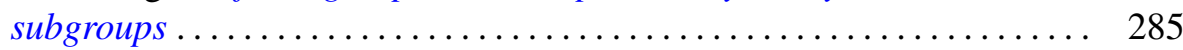

James A. Huckaba, Extensions of pseudo-valuations................. 295

S. A. Huq, Semivarieties and subfunctors of the identity functor ........ 303

I. Martin (Irving) Isaacs and Donald Steven Passman, Finite groups with small character degrees and large prime divisors. II ............ 311

Carl Kallina, A Green's function approach to perturbations of periodic

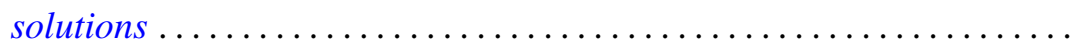

$\mathrm{Al}$ (Allen Frederick) Kelley, Jr., Analytic two-dimensional subcenter manifolds for systems with an integral ....................

Alistair H. Lachlan, Initial segments of one-one degrees ............ 351

Marion-Josephine Lim, Rank k Grassmann products ............. 367

Raymond J. McGivney and William Henry Ruckle, Multiplier algebras of

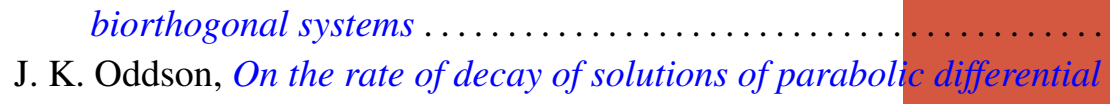

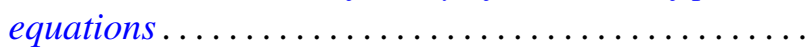

Helmut R. Salzmann, Geometries on surfaces ........... .

Annemarie Schlette, Artinian, almost abelian groups and their groups of automorphisms ............................

Edgar Lee Stout, Additional results on modules over polydisc algebras ...

Lajos Tamássy, A characteristic property of the sphere . .

Mark Lawrence Teply, Some aspects of Goldie's torsion theory. ...

Freddie Eugene Tidmore, Extremal structure of star-shaped sets ...

461

Leon Jarome Weill, Unconditional and shrinking bases in locally convex spaces... 